\title{
Factor VIII as a marker of endothelial cells in follicular carcinoma of the thyroid
}

\author{
HR HARACH, B JASANI, ED WILLIAMS \\ From the Department of Pathology, Welsh National School of Medicine, Heath Park, Cardiff CF4 $4 X N$
}

SUMMARY Factor VIII-related antigen is a recognised marker of endothelial cells. A brief immunocytochemical study of its distribution in follicular carcinoma of the thyroid and its value in the recognition of vascular invasion by this tumour has been carried out. Ten cases of follicular carcinoma of the thyroid were studied. In each strong endothelial staining was found in the majority of vessels in the adjacent normal thyroid. Lymphatic endothelium was negative. In eight of the 10 cases the staining of vessels within the tumour was absent or very weak. Staining was also absent in the majority of vessels completely occluded by tumour, but was present in the endothelium of vessels only partly occluded by tumour. It is concluded that factor VIII-related antigen staining has only limited value in the recognition of vascular invasion in follicular carcinoma. The absence of vascular staining in the tumour leads us to suggest that inhibition of factor VIII production by the tumour could be a possible mechanism which facilitates vascular invasion and metastasis.

Factor VIII, an antihaemophilic factor, has three functionally distinct components: a clot-promoting factor, von Willebrand's factor, and a precipitating antigen, also known as factor VIII-related antigen (F VIII RAG).$^{1-3}$ Tissue culture studies have shown that F VIII RAG and von Willebrand's factor are synthesised by vascular endothelial cells, ${ }^{4}$ and immunoelectron microscopy has demonstrated $F$ VIII RAG in human platelets, megakaryocytes, and endothelial cells. ${ }^{5}$ Recently, antibodies to F VIII RAG have been used in formalin-fixed, paraffinembedded tissues to identify endothelial cells, and to establish the endothelial origin of a number of tumours of uncertain histogenesis. Lesions studied have included Kaposi's sarcoma, ${ }^{6-8}$ cerebellar haemangioblastoma, ${ }^{910}$ and haemangiosarcomas. ${ }^{78}$

In thyroid pathology, there are two main areas where the localisation of an endothelial marker such as F VIII RAG might prove to be of diagnostic value - the definition of the entity of haemangioendothelioma of the thyroid, and the recognition of vascular invasion in follicular carcinoma. Malignant haemangioendothelioma is an extremely uncommon tumour of the thyroid, believed by some authors to be a variant of anaplastic carcinoma, and rarely recognised outside endemic goitrous areas. ${ }^{11}$ We have

Accepted for publication 27 April 1983 confined our attention in this brief study to the pos sible value of the use of F VIII RAG as an endothe ial marker in the recognition of vascular invasion in follicular carcinoma of the thyroid.

\section{Material and methods}

Paraffin blocks of formalin-fixed tissues from $10^{3}$ cases of follicular thyroid tumours with evident of suspected vascular invasion were selected. All cases were studied histologically by routine techniques? including haematoxylin and eosin and orcein foro elastic fibres. Immunoperoxidase technique was car $\underline{3}$. ried out to localise F VIII RAG. The immunolocal-oิ isation technique used was a modification of the DNP-hapten sandwich staining (DHSS) techniqueo described by Jasani, Wynford-Thomas and Williams $;{ }^{12}$ the detailed procedure is given elsewhere. ${ }^{12}$ The sections used to localise F VIII RAG were incubated in trypsin to ensure reproducible staining o Preliminary studies showed that this was achieved after $90 \mathrm{~min}$ incubation under standard conditions. N The dinitrophenylated antiserum to F VIII RAGO was used at a fixed dilution of $1 / 400$. Positive control sections of lymph node and toxic diffuse goitre were included with each batch of staining for F VIIIs? RAG, as were negative controls in which non- 0 immune serum replaced the specific serum. 


\section{Results}

\section{ROUTINE HISTOPATHOLOGY}

All 10 tumours studied had been diagnosed as follicular carcinomas because vascular invasion was considered to be present on haematoxylin and eosin stained sections, confirmed in some but not all cases by elastic techniques. The vascular invasion was present in extracapsular or capsular veins. Seven of the tumours were well differentiated, with a largely follicular pattern, three were less well differentiated, with a largely trabecular or solid pattern. The orcein technique stained the elastic of vessels outside the capsule, and allowed unequivocal identification of their nature. Vessels within or closely applied to the capsule were more difficult to identify, as their elastic structure was sometimes lost in extensive capsular elastic proliferation. Similarly, large vessel invasion by tumour well outside the capsule was easy to identify with certainty, while it was often more difficult to be certain whether rounded masses of tumour in the capsule were within vessels.

\section{IMMUNOLOCALISATION}

Positive staining for F VIII RAG was present in endothelial cells of blood vessels in control sections and in uninvolved thyroid tissue. In general, capillary endothelial cells showed the strongest positivity. As the diameter of vessels increased, the positive staining was weaker. This applied to both arteries and veins. The lining cells of normal lymphatic vessels were negative.

Endothelial cells of vessels within the follicular carcinoma were commonly negative, contrasting markedly with the positivity of endothelial cells of vessels of a similar size in the adjacent nonneoplastic thyroid tissue (Fig. 1). In only two of the 10 tumours was the overall staining of tumour vessels approximately equal to that of the vessels in the surrounding non-neoplastic thyroid. The vessels within the other eight tumours showed negative staining of the endothelium but in three of the tumours very occasional intratumoral vessels were positive.

When presumed vascular lumina outside the tumour were completely occluded by tumour, F VIII RAG staining was negative (Fig. 2), whether or not the structure could be identified as a vessel with elastic stains. In some vessels no endothelial-like cells could be identified, in others residual elongated cells with the morphology and location of endothelial cells were present, but were negative with F VIII RAG. When presumed vascular lumina, seen in the haematoxylin and eosin, were partially occluded by tumour factor VIII RAG staining was positive

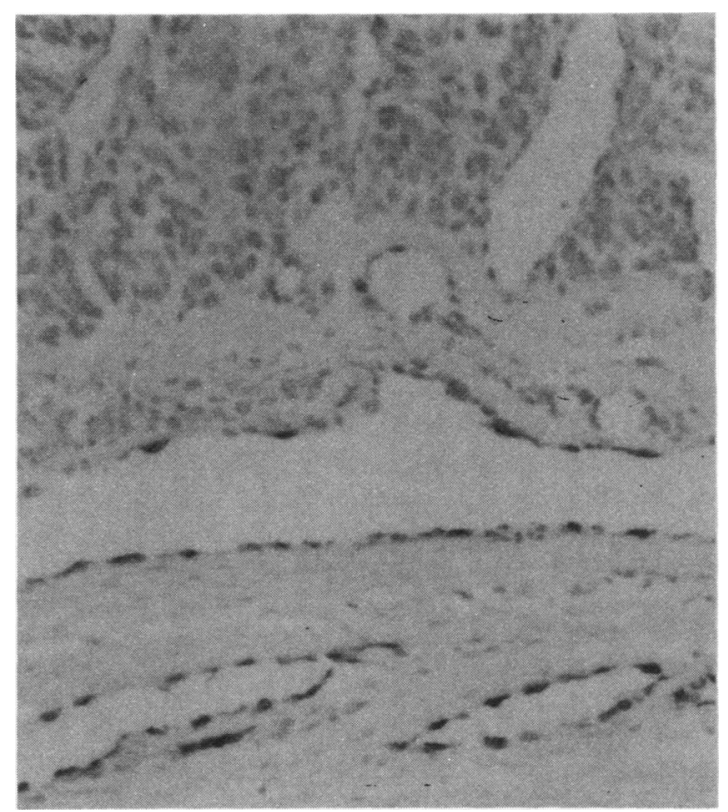

Fig. 1 Follicular carcinoma of thyroid and adjacent normal gland stained for factor VIII RAG. The positive endothelial staining of the vessels outside the capsule (bottom) contrasts strongly with the absence of staining in the tumour vessels (top). DNP-hapten sandwich stain $\times 153$.

whether or not the structure could be identified as a vessel by elastic stains (Fig. 3).

\section{Discussion}

Several studies have stressed the value of the identification of extracapsular vascular invasion in the assessment both of the diagnosis and of the likelihood of distant metastasis in follicular carcinomas. ${ }^{14-16}$ While the recognition of such vascular invasion is sometimes easy, on other occasions it may be debatable. Factor VIII RAG has been shown to be a reliable endothelial marker in normal tissues in a number of studies, ${ }^{1017}$ and has also been used to identify the vascular origin of various tumours. We have therefore in this study investigated the value of F VIII RAG immunolocalisation in the identification of vascular invasion in follicular carcinoma of the thyroid.

Our first finding, that the endothelial cells within thyroid tumours stained less well than the endothelial cells of vessels of similar calibre outside, was unexpected, although variable results had previously been found in endothelium from vessels within 


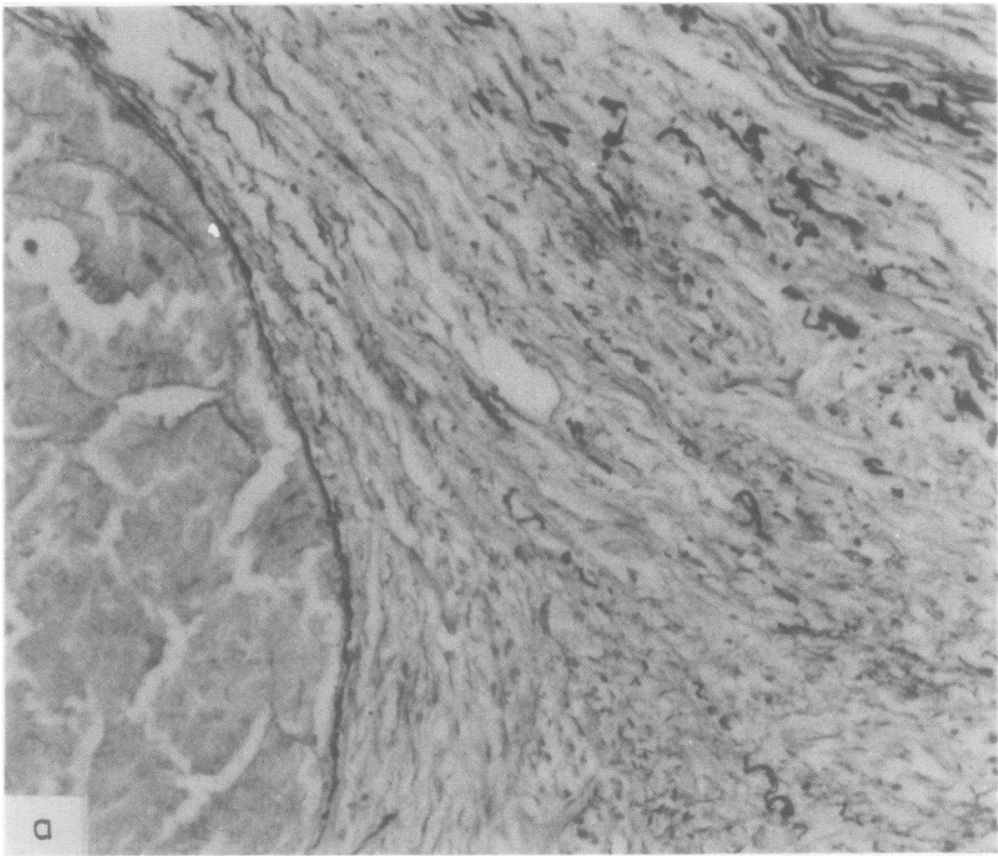

Fig. 2 (a) Vascular invasion by follicular carcinoma, showing residual elastic in the wall. Orcein $\times$ 175. (b) Serial section to (a) stained for factor VIII RAG. No $8 \overrightarrow{0}$ endothelial staining is seen in the

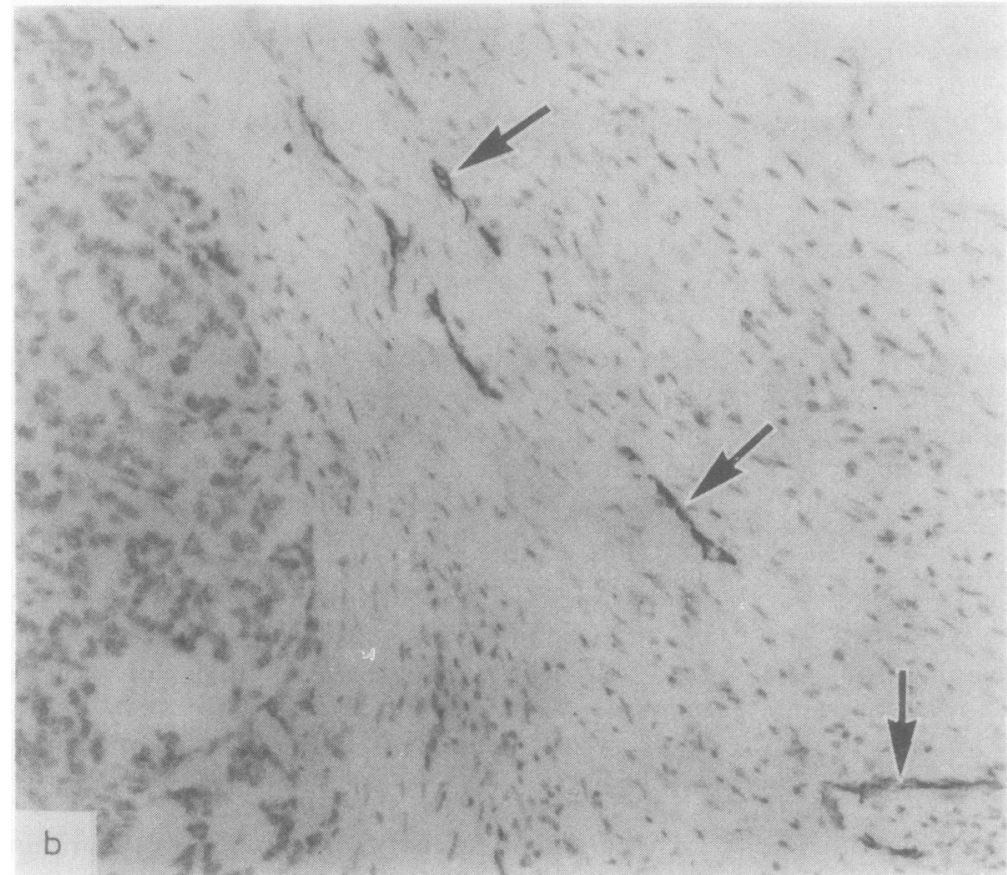
vessel occluded by tumour, compressed capillaries (arrowed) in the adjacent stroma show endothelial positivity. DNP-hapten sandwich stain $\times 175$. 

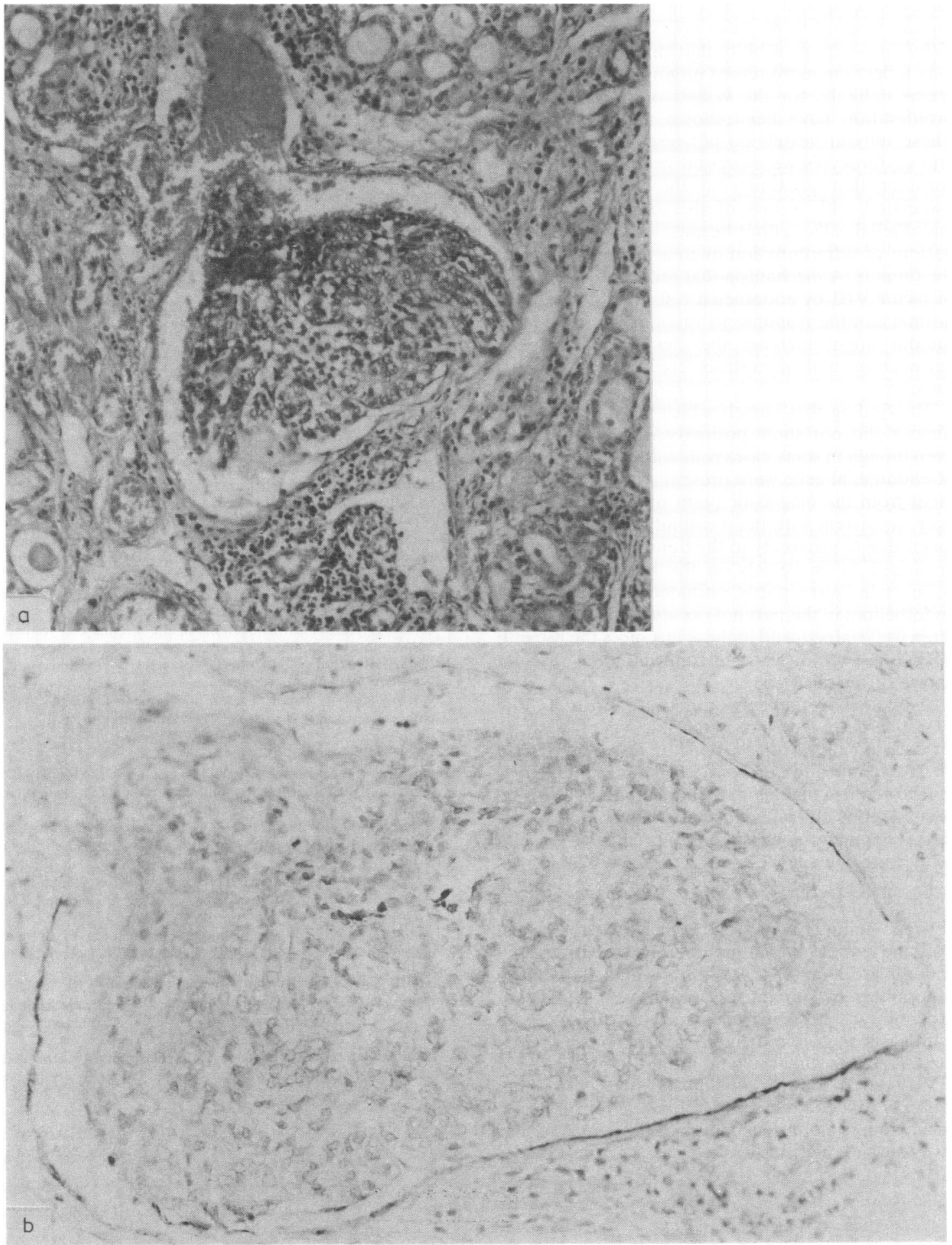

Fig. 3 (a) Small vessel partially occluded by follicular carcinoma and surrounded by normal thyroid tissue. Haematoxylin and eosin $\times 132$. (b) The same vessel as (a) stained for factor VIII RAG and viewed at higher power. Most endothelial cells are positive in this vessel, which lacked demonstrable elastic. DNP-hapten sandwich stain $\times 280$. 
tumours compared to those in the normal background tissue..$^{710}$ We have no explanation for this difference-the possibility that this lack of staining may reflect the more recent origin of tumour vessels seems unlikely, for the vessels of reactive dermal proliferation have been shown to be positive. ${ }^{810}$ These tumour vessels do not resemble lymphatics, and erythrocytes were present in their lumina. We consider that proximity to the tumour may be associated with a loss of the antigen within the endothelial cells-either because of a change in the pattern of differentiation or to a humoral effect by the tumour. A mechanism that reduces the synthesis of factor VIII by endothelial cells, and disturbs the normal clotting response to vascular penetration by tumour might increase the chance that vascular permeation and metastasis could occur in that tumour.

The finding that vessels which were occluded by tumour did not show positivity for F VIII RAG, even though in some cases cells with the morphology of endothelial cells were present, was a disappointment from the diagnostic point of view. It is, however, consistent with the possibility that this antigen loss is due to proximity to the tumour. When spaces, presumed to be vessels on the haematoxylin and eosin stain, were partly occluded by tumour, the endothelium in the part not occupied by tumour was often well stained, and in these instances the use of $F$ VIII RAG was of value in confirming the vascular nature of the structure.

We conclude that immunolocalisation of F VIII RAG has a limited place in the assessment of vascular invasion in follicular carcinoma of the thyroid. In many examples the use of an elastic technique was able to confirm that a major extracapsular vessel had been invaded. In a few instances the use of F VIII RAG immunolocalisation provided useful confirmatory evidence when a vessel was only partially occluded. However, if no elastic layer could be identified, it was still difficult to make the distinction between tumour which had penetrated the vessel wall and tumour which had merely invaginated the endothelial lining of a very thin walled vessel. The generally poor staining in tumour vessels in close proximity to tumour cells, and the reduction in staining in the endothelium of vessels occluded by tumour suggest that the tumour may induce a loss of F VIII RAG from endothelium. It is suggested that this may reflect a reduction in factor VIII synthesis, which represents a mechanism facilitating vascular invasion and metastasis.
We are grateful to Dr JC Giddings of the Depart- $\frac{0}{F}$ ment of Haematology of the Welsh National Schoolo of Medicine for the antibody to factor VIII RAG.

\section{References}

${ }^{1}$ McKee PA, Anderson JC, Switzer ME. Molecular structural studies of human factor VIII. Ann NY Acad Sci 1975;240:833.

2 Jaffe EA. Endothelial cells and the biology of Factor VIII. $N^{\circ}$ Engl J Med 1977;296:377-83.

${ }^{3}$ Ratnoff OD. Antihemophilic factor (factor VIII). Ann Intern ${ }_{\sigma}^{\omega}$ Med 1978;88:403-9.

4 Jaffe EA, Hoyer LW, Nachman RL. Synthesis of antihemophilico antigen by cultured human endothelial cells. J Clin Invest 1973;52:2757-64.

$s$ Piovella F, Nalli G, Malamoni GD, Majolino I, Frassoni F, Sitar ${ }^{\circ}$ GM, et al. The ultrastructural localisation of Factor VIII $\vec{\circ}$ antigen in human platelets, megakaryocytes and endothelial cells utilising a ferritin-labelled antibody. Br J Haematol $\mathrm{O}$ 1978;39:209-13.

- Guarda LG, Silva EG, Ondoñez NG, Smith JL. Factor VIII inKaposi's sarcoma. Am J Clin Pathol 1981;76:197-200.

' Schested M, Hou-Jensen K. Factor VIII-related antigen as ano endothelial cell marker in benign and malignant diseases. Vir- $-\mathbb{D}$ chows Arch [Pathol Anat] 1981;391:217-25.

- Burgdorf WHC, Mukai K, Rosai J. Immunohistochemica identification of Factor VIII-related antigen in endothelia $\Gamma$ cells of cutaneous lesions of alleged vascular nature. Am J Clinco Pathol 1981;75:167-71.

- Jurco S, Nadji M, Harvey DG, Parker JC, Font RL, Morales Hemangioblastomas: histogenesis of the stromal cell studied by immunocytochemistry. Hum Pathol 1982;13:13-8.

${ }^{10}$ McComb RD, Jones TR, Pizzo SV, Bigner DD. Specificity and sensitivity of immunohistochemical detection of Factor VIII/ von Willebrand factor antigen in formalin-fixed parafin-0 embedded tissue. J Histochem Cytochem 1982;30:371-7.

"Hedinger Chr. Geographic pathology of thyroid disease. Pathol Res Pract 1981;171:285-92.

12 Jasani B, Wynford-Thomas D, Williams ED. Use of monoclonaP antihapten antibodies for immunolocalisation of tissue anti gens. J Clin Pathol 1981;34:1000-2.

${ }^{13}$ Harach HR, Williams ED. Fibrous thyroiditis-an immunopathological study. Histopathology-in press.

14 Woolner LB, Beahrs OH, Marden-Black B, McConahey WM:Keating FR. Classification and prognosis of thyroid carcinoma 3 A study of 885 cases observed in a thirty year period. $A m$ y. Surg 1961;102:354-87.

15 Franssila K. Value of the histologic classification of thyroio cancer. Acta Pathol Microbiol Scand [A] 1971;suppl 225 음

${ }^{16}$ Cady B, Sedgewick CE, Meissner WA, Bookwalter JR, Romag7 osa V, Werber J. Changing clinical, pathologic, therapeutie and survival patterns in differentiated thyroid carcinoma. $A n \bar{\pi}$ Surg 1978;184:541-53.

17 Mukai K, Rosai J, Burgdorf WHC. Localisation of Factor VIII related antigen in vascular endothelial cells using a0 immunoperoxidase method. Am J Surg Pathol 1980;4:273-6 స్

Requests for reprints to: Professor ED Williams, Depart ment of Pathology, Welsh National School of Medicin Heath Park, Cardiff CF4 4XN, Wales. 